\title{
A pilot study of the effect of ezetimibe for postprandial hyperlipidemia: Erratum
}

In the article, "A pilot study of the effect of ezetimibe for postprandial hyperlipidemia" ${ }^{[1]}$ which appeared in Volume 97 , Issue 46 of Medicine, En-Zhong Xue's name appeared incorrectly as E-Zhong Xue. The corresponding author information should be Chun-Li Liu, Department of Cardiology, The People's Hospital of Yan'an, No. 57 Qilipu St, Baota Qu, Yan'an 716000, China (E-mail: chunliliu007@126.com).

\section{Reference}

[1] Xue E, Zhang M, Liu C. A pilot study of the effect of ezetimibe for postprandial hyperlipidemia. Medicine. 97;46:e12960. 